Batista, M.; Leyton, M.; Lobato, S.; Jiménez, R. (2019) Transcontextual Model of Motivation in the Preaching of Healthy Lifestyles. Revista Internacional de Medicina y Ciencias de la Actividad $\begin{array}{llllllll}\text { Física y el } & \text { Deporte } & \text { vol. } & 19 & (75) & \text { pp. } & 463-488\end{array}$ Http://cdeporte.rediris.es/revista/revista75/artmodelo1047.htm

DOI: $10.15366 /$ rimcafd2019.75.006

\title{
ORIGINAL
}

\section{MODELO TRANSCONTEXTUAL DE LA MOTIVACIÓN EN LA PREDICCIÓN DE ESTILOS DE VIDA SALUDABLES}

\section{TRANSCONTEXTUAL MODEL OF MOTIVATION IN THE PREACHING OF HEALTHY LIFESTYLES}

\author{
Batista, M. ${ }^{1}$; Leyton, M. ${ }^{2}$; Lobato, S. $^{3}$ y Jiménez, R. ${ }^{4}$ \\ 1 Doctor en Ciencias de la Educación. Profesor adjunto en el Instituto Politécnico de Castelo \\ Branco, SHERU (Sport, Health and Exercise Research Unit) (Portugal) marco.batista@ipcb.pt \\ 2 Doctora en Ciencias de la Actividad Física y del Deporte. Profesora Ayudante Doctora en la \\ Universidad Rey Juan Carlos de Madrid (España) martaleyton1985@gmail.com \\ ${ }^{3}$ Doctora en Ciencias de la Actividad Física y del Deporte. Universidad de Extremadura \\ (España) susanalobato@gmail.com \\ 4 Profesora Titular en la Facultad de Ciencias del Deporte. Universidad de Extremadura \\ (España) ruthji@unex.es
}

Código UNESCO / UNESCO code: 3212 Salud Pública / Public Health, 3206. Clasificación Consejo de Europa / Council of Europe classification: 15. Psicología del Deporte / Sports Psychology

Recibido 5 de octubre de 2017 Received October 5, 2017

Aceptado 7 de abril de 2018 Accepted April 7, 2018

\section{RESUMEN}

El presente trabajo examinó la aplicación del Modelo Transcontextual de la Motivación en la predicción de estilos de vida saludables de atletas veteranos. Se utilizó una muestra de 682 atletas veteranos portugueses de ambos géneros, de edades comprendidas entre los 30 y los 76 años $(M=43.64 ; D T=8.25)$, dónde a través de cuestionarios se ha medido: la satisfacción de las necesidades psicológicas básicas, la motivación, las variables del comportamiento planeado y los estilos de vida saludables. De las conclusiones alcanzadas en este trabajo, son de destacar la relevancia de fomentar la necesidad psicológica básica de relación social, ya que ésta favorecerá la motivación intrínseca, promoviendo un mayor control del comportamiento sobre las intenciones de los practicantes, generando así mejores hábitos alimenticios, hábitos de descanso y menor consumo de tabaco. 
PALABRAS CLAVE: Teoría de la acción planeada, teoría de la autodeterminación, modelo de ecuaciones estructurales, deporte, veteranos.

\section{ABSTRACT}

The present paper has examined the application of the Transcontextual Model of motivation in the prediction of healthy lifestyles of veteran athletes. A sample of 682 Portuguese veteran athletes of both sexes, aged between 30 and 76 years $(M=43.64 ; S D=8.25)$, were administered the following questionnaires: satisfaction of needs basic psychological, self-determination motivation, planned behavioral variables and healthy lifestyles. From the conclusions reached in this work, it is important to emphasize the importance of fostering the basic psychological need of relatedness, since this will favor the intrinsic motivation, promoting greater control of behavior over the intentions of practitioners, thus generating more healthy eating habits, rest habits and lower tobacco consumption.

KEYWORDS: theory of planned behaviour, theory of self-determination, model of structural equations, sport, veterans.

\section{INTRODUCCIÓN}

El deporte se presenta como un contexto socialmente implantado en la globalidad de los países. Es por excelencia un medio educativo y socializador, que confiere a los practicantes desde temprana edad hasta veteranos, todo un conjunto de valores éticos y morales que pueden moldear la personalidad, la forma de estar, la toma de decisión o incluso la ambición personal (Carvalho, Avelar Rosa y Carvalho, 2014). Esta ambición de participación deportiva puede, en muchos casos, generar profesionales en diversas áreas, siendo el deporte una de ellas.

Este estudio se centra esencialmente en el contexto del deporte veterano, utilizando como marco teórico el Modelo Transcontextual de la Motivación (Hagger y Chatzisarantis, 2012). Nuestra sociedad está en constante cambio, observando en las últimas décadas un aumento en la esperanza de vida, acompañado de un aumento del porcentaje de individuos, por encima de los 35 años de edad, que participan en actividades físicas de carácter competitivo y no competitivo (Silva, Dias y Fonseca, 2011; Batista, Jiménez, Leyton, Aspano y Lobato, 2017b).

A pesar de los beneficios reconocidos que supone la realización de actividad física regular para la salud, el porcentaje de individuos que realizan una práctica de actividad física suficiente para conferir beneficios para la salud, es bajo (WHO, 2016). Son conocidos los efectos degenerativos del proceso de envejecimiento del ser humano, descritos con detalle por la American College of Sport Medicine (ACSM, 2010; Riebe, Franklin, Thompson, Garber, Carol, Whitfield, Magal y Pescatello, 2015). 
En cuanto al deporte veterano, corresponde al deporte practicado por atletas que superan los 35 años de edad y continúan disputando pruebas deportivas, aunque en muchas modalidades el término utilizado para clasificar a estos atletas es "atletas masters". Sin embargo, podemos encontrar escalones de participación en clases veteranas desde edades menores, como por ejemplo la natación, desde los 28 años, o en otras modalidades, a partir de los 30 años, como es el caso del triatlón o judo. La participación veterana en el deporte en Portugal, ha evolucionado en número de efectivos federados, señalando un crecimiento desde 2002 a 2009 de un 150\%, a diferencia de los más jóvenes, que sólo duplicaron, el ratio de participación veterana (Instituto do Desporto de Portugal, 2011).

Es relevante indicar que los atletas veteranos, por norma, entrenan y compiten durante prácticamente toda su vida (Baker, Horton, y Weir, 2010), convirtiéndose la motivación en un factor para retardar el envejecimiento y tener una mayor calidad de vida (Zarauz-Sancho y Ruiz-Juan, 2013; Zarauz-Sancho y Ruiz-Juan, 2015; Batista, Jiménez, Lobato, Leyton y Aspano, 2017a).

Esta mayor o menor longevidad competitiva de los atletas, profesionales o aficionados, nos arrastra hacia un campo de reflexión que se relaciona con la calidad de vida, muy vinculada con el bienestar. La noción de bienestar depende de muchos aspectos de la salud, pero igualmente depende de aspectos psicológicos como la autoestima, la percepción de éxito, los objetivos de meta, o los niveles de realización, productividad y desempeño del atleta, siendo la satisfacción con la vida uno de los principales indicadores de bienestar subjetivo (Diener, 2013), contribuyendo a un estilo de vida saludable (Batista, Jiménez, Lobato, Leyton y Aspano (2017a). Es de vital importancia que los ex atletas, muchos de ellos actuales atletas veteranos, sigan encontrando en el deporte una dimensión contextual que les proporcione un estilo de vida saludable y elevada satisfacción con la vida.

El Modelo Transcontextual (Hagger y Chatzisarantis, 2012) nos sugiere una contribución original al conocimiento, ilustrando el comportamiento de los atletas veteranos, a través de la Teoría de la Autodeterminación (Deci y Ryan, 1980, 1985, 1991, 2000), el Modelo Jerárquico de la Motivación (Vallerand, 2007), así como la Teoría del Comportamiento Planeado (Ajzen, 1985), buscando predecir comportamientos de estilos de vida saludables, demostrándolo a través de un modelo de ecuaciones estructurales.

El enfoque del Modelo Transcontextual se centra en explicar el proceso por el cual la motivación en el campo educacional puede ser transferida para contextos externos (Hagger y Chatzisarantis, 2012). Este modelo ha sido ampliamente aplicado en contextos de Educación Física, analizando cómo el apoyo percibido para la motivación en el aula puede influenciar la motivación para la realización de actividad física durante la clase, así como, los comportamientos de ocio evidenciados en la práctica de actividad física real fuera de la escuela (Hagger, Chatzisarantis, Culverhouse y Biddle, 2003; Hagger y Chatzisarantis, 2012, 2016; González-Cutre, Sicilia, Beas-Jiménez y Hagger, 2014). La fuerza de este modelo reside en la integración de diferentes teorías motivacionales (Orbell et al., 2006; Hagger y Chatzisarantis, 2009a), de modo que una explicación es un 
complemento de los procesos motivacionales que son inexplicables de manera aislada (Hagger et al., 2003; Hagger y Chatzisarantis, 2009b).

La Teoría de la Autodeterminación (Deci y Ryan, 1980, 1985, 1991, 2000) explica que la motivación es un continuo caracterizado por distintos niveles de autodeterminación que, de mayor a menor, son la motivación intrínseca, la motivación extrínseca y la desmotivación. Han considerado, por lo tanto, que la dimensión más interna de la motivación era una motivación autónoma para el agente implicado, mientras que la dimensión más externa de la motivación la convertía en una motivación controlada.

Más recientemente, Deci y Ryan (2012) y Vallerand (2015), definen la Teoría de la Autodeterminación como una teoría empírica de la motivación humana y de la personalidad en los contextos sociales, que distingue entre motivación autodeterminada y no autodeterminada. Estos tipos de autodeterminación se concretan en desmotivación (ausencia de intención de actuar), regulación externa, introyectada e identificada (determinada por recompensas y/o agentes externos) y regulación intrínseca (placer al realizar una actividad).

De acuerdo con Ryan (1995), la Teoría de la Autodeterminación establece tres necesidades para el crecimiento psicológico y bienestar, que son: la necesidad psicológica básica de autonomía, competencia y relación social, vistas como el requisito previo para el funcionamiento ideal de esos procesos integrativos del organismo.

Comenzando por caracterizar las tres necesidades psicológicas básicas, la autonomía es la capacidad de escoger lo que parece ser la decisión más adecuada sin cualquier presión externa (Moreno-Murcia, Marzo, MartínezGalindo y Marín, 2011), la capacidad para iniciar tareas o tomar decisiones, el control volitivo y asumir las consecuencias del propio comportamiento (Simões y Alarcão, 2013). Según Deci y Ryan (2000), varios estudios sobre la percepción de autonomía revelan que está relacionada con una mayor motivación intrínseca, mayor satisfacción, y mayor bienestar.

A su vez, la necesidad de percepción de competencia engloba, desde la búsqueda por la supervivencia, la ejecución de actividades prácticas, la exploración del ambiente, hasta la competencia en una participación social efectiva (Deci y Ryan, 2000; Appel-Silva, Wendt y Argimon, 2010). Simplificando, es la capacidad de ejecutar acciones con la certeza de que el resultado es aquel que es lo esperado o deseado (Moreno-Murcia et al., 2011), refiriéndose a un sentimiento personal de eficacia (Simões y Alarcão, 2013).

Por último, la necesidad de relación social, hace referencia a la relación con otras personas, grupos o comunidades, en busca de la actividad de amar y ser amado (Deci y Ryan, 2000; Appel-Silva, et al., 2010). Esta necesidad es el sentimiento de que se puede contar con la colaboración y aceptación de las personas consideradas importantes (Moreno-Murcia et al., 2011) y es esencial para la adquisición de las reglas sociales (normas, reglas y valores), pues es por los vínculos con los otros como ocurre el aprendizaje (Deci y Ryan, 2000). 
Vallerand (1997, 2001), basado en la Teoría de la Autodeterminación propone el Modelo Jerárquico de la Motivación, basado en cuatro pilares fundamentales: niveles jerárquicos de generalidad, factores sociales, el triple constructo de la motivación descrito anteriormente y las consecuencias motivacionales.

De este modo, Vallerand (2007) detalla tres niveles jerárquicos de generalidad en los que se van a desarrollar los diferentes tipos de motivación: global, contextual y situacional. Para este autor, la motivación a nivel global refleja una disposición generalizada de una persona a tener motivación más o menos autodeterminada. La motivación a nivel contextual es una motivación para involucrarse en comportamientos en un determinado contexto, tal como educación física, actividad física de ocio, o competitiva. La motivación en el nivel situacional se refiere a la regulación motivacional en un momento dado, por ejemplo la competición. Siendo en el nivel contextual donde ocurre la transferencia entre los contextos y dónde desarrollamos el presente estudio.

En segundo lugar, los factores determinantes de la motivación se refieren a los factores humanos y no humanos encontrados en el ambiente social, diferenciados también según el nivel de generalidad (Vallerand, 1997).

$Y$ las consecuencias motivacionales pueden ser de tipo afectivo, cognitivo y comportamental (Vallerand, 1997). Por lo tanto, los aspectos sociales del entorno, influyen en la motivación en función de la satisfacción de las necesidades psicológicas básicas, de manera que una mayor satisfacción de las necesidades psicológicas básicas provocará mayores niveles de motivación autodeterminada, originándose consecuencias positivas a nivel afectivo, cognitivo y comportamental (Vallerand, 2001).

Por otro lado, el Modelo Transcontextual aplica los postulados de la Teoría del Comportamiento Planeado (Ajzen, 1985), para explicar cómo los tipos de motivación autodeterminada forman la base social-cognitiva de los juicios sobre futuros comportamientos situacionales. Consecuentemente, el Modelo Transcontextual propone que la motivación intrínseca para la actividad física está relacionada con actitudes, normas subjetivas, y percepción de control percibido sobre el comportamiento (Hagger et al, 2003; Hagger y Chatzisarantis, 2012, 2016). A partir de la teoría de Ajzen $(1985,2014)$, las actitudes, normas subjetivas y percepción de control o comportamental están relacionados con las intenciones que reflejan la cantidad de esfuerzo o intensidad que un individuo va a invertir para perseguir los resultados futuros, de involucrarse por ejemplo en actividades físicas, o participar en competiciones.

Gurtner, Gulfi, Genoud, Trindade, y Schunacher (2012) han descubierto que los individuos tienden a alinear sus niveles de motivación, particularmente adaptables, a través de contextos semejantes, generando "efectos transcontextuales". Este alineamiento y proceso de transferencia también ha sido reconocido y soportado empíricamente en otras áreas (Mata et al., 2009; Pavey y Sparks, 2010; Gurtner et al., 2012). 
Se busca destacar la base conceptual del Modelo Transcontextual y proporcionar detalles de cómo aplicar este modelo en el presente estudio, incorporando las necesidades psicológicas básicas, y teniendo como consecuencia comportamientos de estilos de vida saludables en atletas veteranos.

El constructo de estilo de vida saludable se constituye como un obstáculo a la enfermedad (Odgen y Carrol, 2010; Telama, et al., 2014) e incluye la salud preventiva, la buena nutrición y control del peso, el ocio, la actividad física regular, los períodos de reposo y de relajación, la capacidad para enfrentar condiciones o situaciones adversas y establecer relaciones afectivas solidarias y ciudadanas, adoptando una postura de ser y de estar en el mundo con un objetivo de vivir bien (WHO, 2002).

El estudio de los estilos de vida saludables puede ser marcado hasta la actualidad por tres grandes períodos: un primer período con inicio en el siglo XIX, hasta mediados del siglo XX; un según período, que encierra la segunda mitad del siglo XX y un tercer período que se centra en la actualidad. Durante el primer período, se destacan estudios (Veblen, 1994; Adler, 1929; Weber, 1946) que realzan los estilos de vida saludable, dependientes de una visión sociológica y de factores individuales de los individuos que integran los estratos sociales que los pueden mantener. En el segundo período, se destacaron estudios (Bandura, 1982; Rodríguez-Marín y García-Hurtado, 1995) que relatan una incorporación de los estilos de vida saludables en el área de la salud y estudios referentes a conductas aisladas. En el tercer período, ha habido un desarrollo hasta la actualidad, donde hay que destacar trabajos (Prochaska, Spring y Nigg, 2008; García-Ubaque, 2011) que reflejan conductas saludables integradas en contextos diversos, o, la relación de diferentes dimensiones con los estilos de vida saludables, tal como la motivación o las necesidades psicológicas básicas, procurando entender la autodeterminación para una determinada práctica y la adopción de conductas saludables.

A continuación presentamos algunos estudios que investigaron las mismas variables integrantes del Modelo Transcontextual y estilos de vida saludables, que proponemos en nuestro estudio.

Son conocidos los efectos degenerativos del proceso de envejecimiento del ser humano, descritos detalladamente por el American College of Sport Medicine (ACSM, 2010). Sin embargo, resulta muy interesante observar que los atletas veteranos por norma, entrenan y compiten durante prácticamente toda su vida (Baker, Horton, y Weir, 2010), por lo que la motivación por retrasar el envejecimiento y tener una mayor calidad de vida, podría ser una de las más altas motivaciones de este tipo de atletas, según indicadores obtenidos por Zarauz-Sancho y Ruiz-Juan (2013, 2015, 2016).

Algunos trabajos (Moreno, Moreno y Cervelló, 2007; DeFreese y Smith, 2013) han mostrado la relación entre la práctica de actividades físico-deportivas y la motivación más autodeterminada. En el caso de los atletas veteranos, también la motivación debe ser considerada un determinante psicológico que puede interferir en el rendimiento deportivo del atleta. Según Da Silva (2009) y Zarauz- 
Sancho y Ruiz-Juan (2015), las motivaciones de estos atletas son principalmente intrínsecas, aunque las motivaciones extrínsecas, los motivos de salud, el gusto por la práctica, las relaciones sociales y la propia competición también sean muy importantes.

Hodge, Allen, y Smellie (2008) han analizado practicantes de seis deportes diferentes, con edades comprendidas entre los 29 y 77 años, donde han observado que estos presentaban una percepción elevada de su capacidad y mayoritariamente evidenciaban una motivación intrínseca, independientemente del tipo de deporte.

Estudiando específicamente atletas veteranos que entrenaban y competían habitualmente en atletismo, Da Silva (2009) ha encontrado que sus motivaciones eran fundamentalmente intrínsecas, aunque también tuviesen importancia las motivaciones extrínsecas, los motivos de salud, las relaciones sociales y la competición.

En estudios inherentes al grado de autodeterminación en función de una serie de hábitos de entrenamiento y del historial atlético, los atletas veteranos han revelado elevados niveles de motivación intrínseca, moderados niveles de motivación extrínseca y casi nulos de desmotivación (Ruiz-Juan y ZarauzSancho, 2012), pues para estos atletas lo más importante de su práctica deportiva es la satisfacción por la superación de sus límites durante los entrenamientos y después, superar a los adversarios en las competiciones, esperando una medalla, o hasta un récord (Sancho y Ruiz-Juan, 2014, 2016).

En un estudio sobre atletas veteranos de judo realizado por Batista, JiménezCastuera, Honório, Petrica y Serrano (2016b), los autores han obtenido niveles elevados en las necesidades psicológicas básicas, con principal atención para la percepción de relaciones sociales, una elevada motivación autónoma, así como valores reducidos del nivel de la motivación controlada y de desmotivación.

En la misma línea de investigación, Batista, Jiménez, Leyton, Aspano y Lobato (2017b), pero recorriendo varias modalidades, han obtenido valores más elevados en la motivación autónoma, particularmente en la dimensión motivación intrínseca, así como en la percepción de la necesidad psicológica básica de relaciones sociales.

Cuando comparamos los géneros en atletas veteranos, Batista, Jiménez, Lobato, Leyton y Aspano (2017a) observaron que no existían diferencias significativas en cuanto a la motivación autodeterminada. No obstante, las atletas femeninas presentaron diferencias significativas a su favor, en la satisfacción de las necesidades psicológicas básicas.

En un estudio de Leyton et al. (2012), donde se utilizó una muestra de mujeres ancianas, se concluyó que los niveles de motivación más autodeterminados presentaban una relación positiva, con las variables de estilos de vida que realzan la salud, tal como los hábitos alimenticios, el descanso, y una relación 
negativa, con la variable de estilos de vida que perjudican la salud, en particular con el consumo de tabaco.

A su vez, en otra investigación (Leyton, 2014) fue utilizada una muestra de 135 mujeres con edades comprendidas entre los 40 y los 88 años para llevar a cabo un programa de ejercicio físico, con educación nutricional y fomento de la motivación intrínseca, con el objetivo de mejorar las variables de estilos de vida que realzan la salud de las personas adultas y ancianas, se comprobó que el fomento de motivación intrínseca favorecía, positivamente, el aumento de la satisfacción de las necesidades psicológicas básicas de autonomía y competencia, las formas de motivación más autodeterminadas y los hábitos alimenticios.

Pizarro (2014) con una muestra de 94 mujeres, con edades comprendidas entre los 42 y los 88 años, determinó que la regulación intrínseca, la regulación integrada y la regulación identificada se relacionaban de forma positiva y significativa con las variables de estilos de vida que realzan la salud (descanso y hábitos alimenticios saludables). La desmotivación se relacionaba de forma positiva, pero, no significativa con la variable de estilo de vida nociva para la salud, consumo de tabaco.

Otro estudio llevado a cabo por Moreno, Hernández y González-Cutre (2009) ha mostrado que la satisfacción de las necesidades de autonomía y relación con los demás predecían, positivamente, la motivación intrínseca. También es necesario mencionar que diversos autores (Ruiz-Juan et al., 2014; Vega et al., 2015) han indicado que el consumo de tabaco es un factor que predispone al consumo de alcohol de manera significativa.

Más recientemente, Batista et al. (2016a) con una muestra de 684 atletas veteranos, entre los 30 y los 90 años, donde se quería relacionar determinantes motivacionales con estilos de vida saludables, han obtenido valores de motivación intrínseca, regulación identificada, regulación integrada, hábitos alimentarios y de descanso bastante elevados. Los valores de regulación externa y consumo de tabaco fueron bastante reducidos. Los resultados han revelado que los factores de hábitos alimenticios y de descanso estaban correlacionados, positivamente, con los factores de motivación intrínseca, regulación integrada, regulación identificada; el factor del consumo de tabaco estaba correlacionado, positivamente, con los factores de regulación introyectada, regulación externa y desmotivación.

La motivación determina el sentido, la intensidad y la persistencia de comportamientos, explicando el por qué las personas hacen o participar en ciertas actividades, y con qué determinación y cuánto tiempo invierten en ellas (Iso-Ahola y St.Clair, 2000). La motivación está íntimamente ligada con la dimensión intencional del comportamiento y es un constructo determinante para analizar el contexto de promoción de la actividad física (Hagger et al., 2007a) particularmente en el deporte veterano (Zarauz-Sancho y Ruiz-Juan, 2015; Batista et al., 2017b). La práctica de actividad física es asumida como un comportamiento intencional que es directamente afectado por antecedentes motivacionales (Hagger y Chatzisarantis, 2009a), donde en esa línea el Modelo 
Transcontextual de la motivación ha sido desarrollado (Hagger et al, 2003; Hagger y Chatzisarantis, 2012; Hagger y Chatzisarantis, 2016). El presente estudio va a analizar si el modelo puede ser replicado en este contexto culturalmente singular, así como realizar una extensión de la Teoría de la Autodeterminación (Deci y Ryan, 1991).

Teniendo en cuenta la revisión y las tendencias expuestas, el presente estudio ha sido diseñado con el objetivo de testar el Modelo Transcontextual de la motivación en la predicción de estilos de vida saludables de atletas veteranos.

\section{MATERIAL Y MÉTODOS}

Este es un estudio transversal que incidirá en determinar el Modelo Transcontextual a través de la aplicación de un modelo de ecuaciones estructurales, atendiendo a las recomendaciones de los autores de referencia (Hu y Bentler, 1999; Schunacker y Lomax, 2010).

\section{Participantes}

La muestra del estudio estuvo compuesta por 682 atletas veteranos portugueses de ambos géneros, de edades comprendidas entre los 30 y los 76 años $(M=43,64 ; D P=8,25)$, de los cuales el $80 \%$ (545 individuos) eran de género masculino y $20 \%$ (137 individuos) de género femenino, competidores de varias modalidades deportivas. Constan en este estudio datos referentes a practicantes de modalidades colectivas, como el fútbol $(27,8 \%)$, hockey patín (3,2\%), rugbi ( $7,3 \%)$, baloncesto $(2,1 \%)$, así como modalidades individuales, en particular, tenis $(9,7 \%)$, judo $(15,2 \%)$, atletismo $(13,2 \%)$, ciclismo/BTT $(9,1 \%)$, triatlón/duatlón (4,4\%), equitación (1,5\%) y natación (2,2\%). El tipo de muestreo utilizado para la selección de la muestra del presente estudio ha sido no aleatorio, una vez que no asienta en una base probabilística, estando inherente a la recolecta de datos una elección intencional de sujetos con determinadas características específicas (Cubo-Delgado, Martín-Marin y Ramos-Sanchez, 2011).

\section{Instrumentos}

A continuación se van a describir las variables y los instrumentos de medida.

- Necesidades Psicológicas Básicas. Para medir la satisfacción de las necesidades psicológicas básicas se ha aplicado la versión portuguesa de la Basic Psychological Needs Exercise Scale (BPNES) (Vlachopoulos y Michailidou, 2006), validado por Pires, Cid, Borrego, Alves y Silva (2010). Esta escala esta constituida por 12 ítems distribuidos en 3 dimensiones que reflejan las necesidades psicológicas básicas de la Teoría de la autodeterminación: autonomía, percepción de competencia y percepción de relaciones sociales, estando cada dimensión compuesta por 4 ítems que pueden ser clasificados teniendo en cuenta una escala del tipo Likert, entre 1 (discrepo totalmente) y 5 (concuerdo totalmente). Moutão, Cid, Alves, Leitão y Vlachopoulos (2012) a través de un análisis confirmatorio han comprobado la adecuación de la 
adaptación efectuada. En el presente estudio, el modelo de medida ha revelado valores aceptables de ajuste: $X 2=50,95, p \leq 0,01, x 2 / g I=4,63, C F I=0,98, N N F I=$ 0,95 , SRMR $=0,03$, RMSEA $=0,02$, obteniéndose los siguientes índices de fiabilidad interna: percepción de autonomía $(\alpha=0,78)$, percepción de competencia $(\alpha=0,71)$, percepción de relación social $(\alpha=0,78)$.

- Motivación. Para medir la motivación se ha aplicado el Behaviour Regulation Sport Questionnaire (BRSQ) Monteiro et al. (2013), a partir del cuestionario de Lonsdale, Hodge y Rose (2008). Este cuestionario es constituido por 24 ítems los cuales se responden en una escala tipo Likert de 7 niveles, que varían entre 1 ("totalmente en desacuerdo") y el 7 ("totalmente de acuerdo"). Los ítems se agrupan, posteriormente, en 6 factores (con 4 ítems cada uno), que reflejan los tipos de motivación subyacente al continuo motivacional de la Teoría de la autodeterminación (SDT) (Deci y Ryan, 1985; 2000). Para el presente estudio se ha utilizado la versión validada de manera preliminar para la población portuguesa (Monteiro, Moutão, Batista y Cid, 2014), con un análisis factorial confirmatorio, en una muestra de 623 atletas de la modalidad de fútbol, presentando su modelo de medida ( 6 factores con 3 ítems cada). En el presente estudio se han obtenido los siguientes valores de ajuste: $x 2=177,366, p \leq 0,01$, $\mathrm{x} 2 / \mathrm{gl}=4,32, \mathrm{CFI}=0,97, \mathrm{NNFl}=0,95, \mathrm{SRMR}=0,04, \mathrm{RMSEA}=0,07$, así como los índices de fiabilidad interna: desmotivación $(\alpha=0,81)$, motivación autónoma ( $\alpha=$ $0,88)$, motivación controlada $(\alpha=0,89)$.

- Comportamiento Planeado. Para medir las variables del comportamiento planeado asociado a la práctica deportiva (intensiones, actitudes, normas subjetivas y percepción de control) se ha aplicado el cuestionario ya aplicado por otros autores (Courneya y Bobick, 2000; Palmeira y Teixeira, 2004; Palmeira, Teixeira, Branco, Martins, Minderico, Barata, Serpa y Sardinha, 2007). Éste está constituido por 17 ítems estándar, validado para portugués por Palmeira y Teixeira (2004), elaborado a partir de las directrices de Ajzen (1991), divididos en cuatro dimensiones, utilizando una escala de tipo Likert de 7 puntos para proceder a su clasificación.

En este estudio, el modelo de medida ha revelado valores aceptables de ajuste: $\mathrm{X} 2=200,68, \mathrm{p} \leq 0,01, \mathrm{X} 2 / \mathrm{gl}=3,40, \mathrm{CF}=0,94, \mathrm{NNFI}=0,92, \mathrm{SRMR}=0,05$, RMSEA $=0,06$, obteniéndose los siguientes índices de fiabilidad interna: actitudes $(\alpha=0,79)$, normas subjetivas $(\alpha=0,79)$, percepción de control $(\alpha=0,73)$, intenciones $(\alpha=0,71)$.

- Estilos de Vida Saludables: Para medir los estilos de vida saludables se utilizó el Cuestionario de Estilos de Vida Saludables (EVS) (Batista, Jiménez, Leyton, Lobato y Aspano (2016a). Este Cuestionario de Estilos de Vida Saludables (EVS) resulta de la versión traducida y adaptada al castellano, del cuestionario diseñado por Wold (HBSC) (1995).

El EVS presenta la versión de 20 ítems, respetivamente, en la medida en que mide los hábitos alimentarios concretamente los hábitos alimentarios respecto a los horarios de las comidas; los hábitos alimentarios respecto a una alimentación equilibrada; el consumo de tabaco y los hábitos de descanso. En este estudio se ha utilizado una escala de tipo Likert que varía entre "totalmente en desacuerdo" 
(1) y "totalmente de acuerdo" (5). En el presente estudio, el modelo de medida ha revelado valores aceptables de ajuste: $X^{2}=632,68, p \leq 0,001, X 2 / g l=2,01$, $\mathrm{CFI}=0,94, \mathrm{IFI}=0,94, \mathrm{GFI}=0,92, \mathrm{SRMR}=0,06, \mathrm{RMSEA}=0,07$, con un índice de fiabilidad interna: hábitos alimenticios $(\alpha=0,80)$; hábitos de descanso $(\alpha=$ $0,84)$ y consumo de tabaco $(\alpha=0,91)$.

\section{Procedimiento}

Se llevó a cabo una selección de centros deportivos, como clubes y asociaciones, atendiendo a una muestra intencional (Cubo Delgado, MartínMarín y Ramos-Sánchez, 2011). Para la recogida de información, nos pusimos en contacto directo con los deportistas veteranos para solicitar su colaboración en el estudio pidiendo, la firma de un consentimiento informado. La administración del cuestionario definitivo, que ha aglutinado las escalas descritas anteriormente se realizó en presencia del investigador principal, para explicar de forma breve los objetivos y estructura, así como la forma de cumplimentación. Durante el proceso, el investigador principal estaba disponible para cualquier problema que pudiese surgir. El tiempo aproximado de cumplimentación fue aproximadamente de treinta minutos.

\section{Análisis de datos}

Se ha procedido a un análisis del supuesto de normalidad a través de la prueba de Kolmogorov Smirnov, obteniendo una distribución normal de los datos en la muestra. En segundo lugar, se ha realizado un análisis descriptivo de correlación de todas las variables de estudio. En tercer lugar, para comprobar la relación existente entre las variables propuestas, se ha aplicado el método de dos pasos propuesto por Anderson y Gerbing (1988). En el primer paso, se ha probado la validez de constructo del modelo de medida a través de un análisis factorial confirmatorio (modelo de medición). Se han dividido los ítems que componían los factores latentes en dos grupos aleatorios, realizando dicho análisis basándonos en las medidas observadas en los constructos latentes que se correlacionan libremente (Anderson y Gerbing, 1988). En un segundo paso, se ha realizado un modelo de ecuaciones estructurales con el que se han analizado las relaciones predictivas entre las variables analizadas. Todos los análisis han sido desarrollados a través de los programas estadísticos SPSS 21.0 y EQS 6.1.

\section{RESULTADOS}

\section{Análisis descriptivos y de fiabilidad de todas las variables}

En la Tabla 1 podemos observar el análisis descriptivo y de fiabilidad de las variables de análisis. Con respecto a las variables de la Teoría de la Autodeterminación, en las necesidades psicológicas básicas, la percepción de la necesidad psicológica básica de relación social ha sido la variable más puntuada, obteniendo una media de 4,31 , la percepción de autonomía ha presentado una media de 4,05 y la percepción de la necesidad psicológica básica de competencia ha obtenido una media de 4,02. En el dominio de la motivación, la motivación intrínseca ha sido la más valorada con una media de 6,07 puntos, la 
regulación integrada ha obtenido una media de 5,40, la regulación identificada 5,45 , la regulación introyectada 2,37 , la regulación externa 1,78 y la desmotivación una media de 2,26.

De las variables de la Teoría del Comportamiento Planeado, las intenciones ha sido la variable más valorada por los atletas veteranos obteniendo un valor medio de 6,21. La variable actitudes ha presentado una media de 6,13, en la variable normas subjetivas se ha obtenido una media de 5,76 y en la percepción de control se ha obtenido una media de 5,60.

En las variables de estilos de vida saludables, los hábitos alimenticios revelaron una media de 3,75, los hábitos de descanso 3,42, y el consumo de tabaco 1,61.

Tabla 1. Estadística descriptiva y análisis de fiabilidad.

\begin{tabular}{lcccccc}
\hline \multicolumn{1}{c}{ Variable } & Escala & Mín. & Máx. & $M$ & $D T$ & $\begin{array}{c}\text { Alfa de } \\
\text { Cronbach }\end{array}$ \\
\hline 1. Percepción de Autonomía & $1-5$ & 2,00 & 5,00 & 4,05 & 0,59 & 0,78 \\
2. Percepción de Competencia & $1-5$ & 2,00 & 5,00 & 4,02 & 0,50 & 0,71 \\
3. Percepción Relación Social & $1-5$ & 1,75 & 5,00 & 4,31 & 0,55 & 0,78 \\
4. Motivación Intrínseca & $1-7$ & 1,00 & 7,00 & 6,07 & 0,88 & 0,81 \\
5. Regulación Integrada & $1-7$ & 1,00 & 7,00 & 5,40 & 1,12 & 0,79 \\
6. Regulación Identificada & $1-7$ & 1,00 & 7,00 & 5,45 & 1,01 & 0,70 \\
7. Regulación Introyectada & $1-7$ & 1,00 & 7,00 & 2,37 & 1,32 & 0,78 \\
8. Regulación Externa & $1-7$ & 1,00 & 7,00 & 1,78 & 1,05 & 0,90 \\
9. Desmotivación & $1-7$ & 1,00 & 7,00 & 2,26 & 1,25 & 0,85 \\
10. Actitudes & $1-7$ & 1,25 & 7,00 & 6,13 & 0,74 & 0,79 \\
11. Normas Subjetivas & $1-7$ & 1,00 & 7,00 & 5,76 & 1,11 & 0,79 \\
12. Percepción Control & $1-7$ & 2,25 & 7,00 & 5,60 & 1,02 & 0,73 \\
13. Intenciones & $1-7$ & 1,00 & 7,00 & 6,21 & 0,97 & 0,70 \\
14. Hábitos Alimenticios & $1-5$ & 1,13 & 5,00 & 3,75 & 0,70 & 0,80 \\
15. Hábitos de Descanso & $1-5$ & 1,00 & 5,00 & 3,42 & 0,97 & 0,84 \\
16. Consumo de Tabaco & $1-5$ & 1,00 & 4,40 & 1,61 & 0,91 & 0,91 \\
\hline
\end{tabular}

En la Tabla 2 observamos el análisis de correlación de Pearson, donde se determinó que las variables percepción de la necesidad psicológica básica de autonomía, percepción de la necesidad psicológica básica de competencia, percepción de la necesidad psicológica básica de relación social, motivación intrínseca, regulación integrada, regulación identificada, actitudes, normas subjetivas, percepción de control, intenciones y hábitos alimenticios y de descanso se correlacionaban positiva y significativamente entre sí. Las variables regulación introyectada, regulación externa y desmotivación han presentado correlaciones negativas y en su mayoría significativas con las demás variables, a excepción de la variable consumo de tabaco, con la cual se han obtenido correlaciones negativas y significativas. 
Rev.int.med.cienc.act.fís.deporte - vol. 19 - número 75 - ISSN: 1577-0354

Tabla 2. Coeficiente de correlación de Pearson entre las variables de estudio.

\begin{tabular}{|c|c|c|c|c|c|c|c|c|c|c|c|c|c|c|c|}
\hline Variable & 2 & 3 & 4 & 5 & 6 & 7 & 8 & 9 & 10 & 11 & 12 & 13 & 14 & 15 & 16 \\
\hline 1. P. Autonomía & $.715^{* *}$ & $.462^{* *}$ & $.337^{* *}$ & $.405^{* *}$ & $277^{\prime \prime}$ & -.002 & $-.203^{* \prime}$ & $-.234^{4 *}$ & $.158^{* \prime}$ & $.208^{* \prime}$ & $.383^{* \prime}$ & $.219^{* *}$ & $.295^{* \prime}$ & $.211^{* *}$ & -.047 \\
\hline 2. P. Competencia & & $.550^{* *}$ & $.339^{* *}$ & $.385^{*}$ & $.309^{* *}$ & .004 & $-.173^{* *}$ & $-.197^{* *}$ & $.217^{* *}$ & $.250^{* *}$ & $.369^{* *}$ & $.247^{* *}$ & $.330^{* *}$ & $279^{* *}$ & -.045 \\
\hline 3. P. Rel. Sociales & & & $.305^{* *}$ & $.341^{* \prime}$ & $.240^{* *}$ & -.071 & $-.225^{* *}$ & $-.254^{* *}$ & $.119^{* *}$ & $.281^{* *}$ & $.180^{* *}$ & $.183^{* *}$ & $.195^{* *}$ & $.156^{* *}$ & -.052 \\
\hline 4. M. Intrínseca & & & & $.594^{* \prime}$ & $.581^{* *}$ & $-.360^{* *}$ & $-.555^{* *}$ & $-.439^{* *}$ & $.166^{* *}$ & $.188^{* *}$ & $.233^{* *}$ & $.127^{* *}$ & $.284^{* *}$ & $.176^{* *}$ & -.034 \\
\hline 5. R. Integrada & & & & & $.636^{* *}$ & -.028 & $-.282^{* *}$ & $-.194^{* *}$ & $.125^{* *}$ & $.255^{*}$ & $.224^{* *}$ & $.216^{* *}$ & $.184^{* *}$ & .073 & -.014 \\
\hline 6. R. Identificada & & & & & & $-.096^{*}$ & $-.328^{* *}$ & $-.279^{* *}$ & $.155^{* *}$ & $.267^{* *}$ & $.103^{* *}$ & $.331^{* *}$ & $.182^{* *}$ & $.172^{* *}$ & .048 \\
\hline 7. R. Introyectada & & & & & & & $.674^{* *}$ & $.498^{* *}$ & $-.095^{*}$ & .054 & $-.121^{* *}$ & -.011 & $-.077^{*}$ & -.071 & $.126^{* *}$ \\
\hline 8. R. Externa & & & & & & & & $.749^{* *}$ & $-.138^{* *}$ & $-.086^{*}$ & $-.235^{*}$ & $-.143^{* *}$ & $-.182^{* *}$ & $-.114^{* *}$ & $.099^{* *}$ \\
\hline 9. Desmotivación & & & & & & & & & $-.232 *$ & -.041 & $.213^{++}$ & $-.173^{* *}$ & $-.164 *$ & $-.118^{* *}$ & $.104^{* *}$ \\
\hline 10. Actitudes & & & & & & & & & & $.151^{*}$ & $.239^{* *}$ & $.217^{*}$ & $.220^{* *}$ & $.168^{* *}$ & -.066 \\
\hline 11. N. Subjetivas & & & & & & & & & & & $.195^{* *}$ & $.306^{* *}$ & $.163^{* *}$ & .040 & -.028 \\
\hline 12. P. Control & & & & & & & & & & & & $.269^{* *}$ & $.428^{* *}$ & $.267^{* *}$ & $-.251^{*}$ \\
\hline 13. Intenciones & & & & & & & & & & & & & $.201^{* *}$ & $.153^{* *}$ & $-.076^{*}$ \\
\hline 14. H. Alimenticios & & & & & & & & & & & & & & $.582^{* *}$ & $.140^{* *}$ \\
\hline 15. H. Descanso & & & & & & & & & & & & & & & $-.092^{*}$ \\
\hline 16. C. Tabaco & & & & & & & & & & & & & & & - \\
\hline
\end{tabular}

Nota. ${ }^{*} P<.05 .{ }^{* *} P<.01$. P.= Percepción; M.= Motivación; R.= Regulación; N.= Normas; H.= Hábitos; C.= Consumo

\section{Modelo de medición}

Para poder realizar la análisis del modelo de medición y probar el modelo de ecuaciones estructurales (MEE), se ha reducido el número de variables latentes por factor. Este proceso se recomienda especialmente cuando el tamaño de la muestra no es particularmente grande comparada con el número de variables del modelo (Marsh, Richards, Jonson, Roche y Tremayne, 1994; Vallerand, 2001, 2007). Esta reducción puede ser efectuada mediante la combinación de los ítems en parejas. Así, la mitad de los primeros tres ítem de cada subescala han sido medidos para formar el primer bloque de ítem, en la segunda mitad dos ítems se ha procedido de la misma forma para el segundo bloque de ítem, y así hasta el último. Marsh et al. (1994) han propuesto el uso de los pares de ítem porque los resultados de estos son más fiables, tienden a ser distribuidos de forma más normal, y porque se ha reducido a mitad el ratio del número de variables medidas en el modelo y el número de los participantes del estudio.

Se ha considerado una serie de coeficientes fit para evaluar la bondad de ajuste de los modelos de medición con los datos empíricos. Así, basándonos en las aportaciones de diferentes autores (Bentler, 1990; Bollen y Long, 1993; McDonald y Marsh, 1990), los índices fit o índices de bondad de ajuste que se han considerado para evaluar la bondad del modelo de medición han sido: $x 2$, X2/gl RMSEA (Root Mean Square Error of Aproximation), RMSR (Root Mean Square Residual) y los índices incrementales (CFI, IFI y TLI). Estos índices de bondad de ajuste son considerados aceptables cuando o $\mathrm{x} 2 / \mathrm{gl}$ es inferior a 5 , los índices incrementales (CFI, IFI y TLI) son superiores a .90 y los índices de error (RMSEA y RMSR) son inferiores a 08 (Browne y Cudeck, 1993; Hu y Bentler, 1999). Los índices obtenidos después de la análisis han sido: $X 2=519.174, p<$ $.001 ; \mathrm{X} 2 \mathrm{gl}=3.04 ; \mathrm{CFI}=.95 ; \mathrm{IFI}=.95 ; \mathrm{TLI}=.94 ; \mathrm{GFI}=.94 ; \mathrm{RMSEA}=.55 ; \mathrm{SRMR}$ $=.41$. También se ha examinado la validez discriminante del modelo, respetando que la correlación entre las variables latentes, atenuadas por el error de medición 
(+/- 2 veces el error de medición), ha sido inferior a 1.0. Por lo tanto, según las indicaciones anteriores, los resultados han mostrado que el modelo de medición era adecuado.

\section{Modelo de ecuaciones estructurales}

Con el objetivo de analizar las relaciones existentes entre las variables pertenecientes al modelo que se planea (percepción de la necesidad psicológica básica de relación social, motivación intrínseca, percepción de control, intenciones, hábitos alimenticios, hábitos de descanso y consumo de tabaco), se ha utilizado el Modelo de Ecuaciones Estructurales. El test de bondad del modelo ha mostrado los siguientes índices de ajuste: $X^{2}=749.060, p<.001, x 2 / g l=$ 4.44, $\mathrm{CFI}=.91 ; \mathrm{IFI}=.91 ; \mathrm{TLI}=.90 ; \mathrm{GFI}=.91 ; \mathrm{RMSEA}=.071 ; \mathrm{SRMR}=.069$. Estos datos se ajustan a los parámetros establecidos, por lo que se puede aceptar como adecuado el modelo propuesto (Hu y Bentler, 1999). De igual modo, la contribución de cada uno de los factores para la predicción de otras variables se ha examinado a través de pesos de regresión estandarizados. El valor "t" asociado a cada peso se ha tomado como una medida de contribución, de modo que valores superiores a 1.96 se consideran como significativos. En la Figura 1 observamos que la percepción de la necesidad psicológica básica de relación social predice positiva y significativamente la motivación intrínseca. A su vez, ésta predice positiva y significativamente la percepción de control, prediciendo ésta, positiva y significativamente las intenciones y negativa $y$ significativamente el consumo de tabaco. Las intenciones predicen positiva y significativamente los hábitos alimenticios y los hábitos de descanso y negativa y significativamente el consumo de tabaco. 


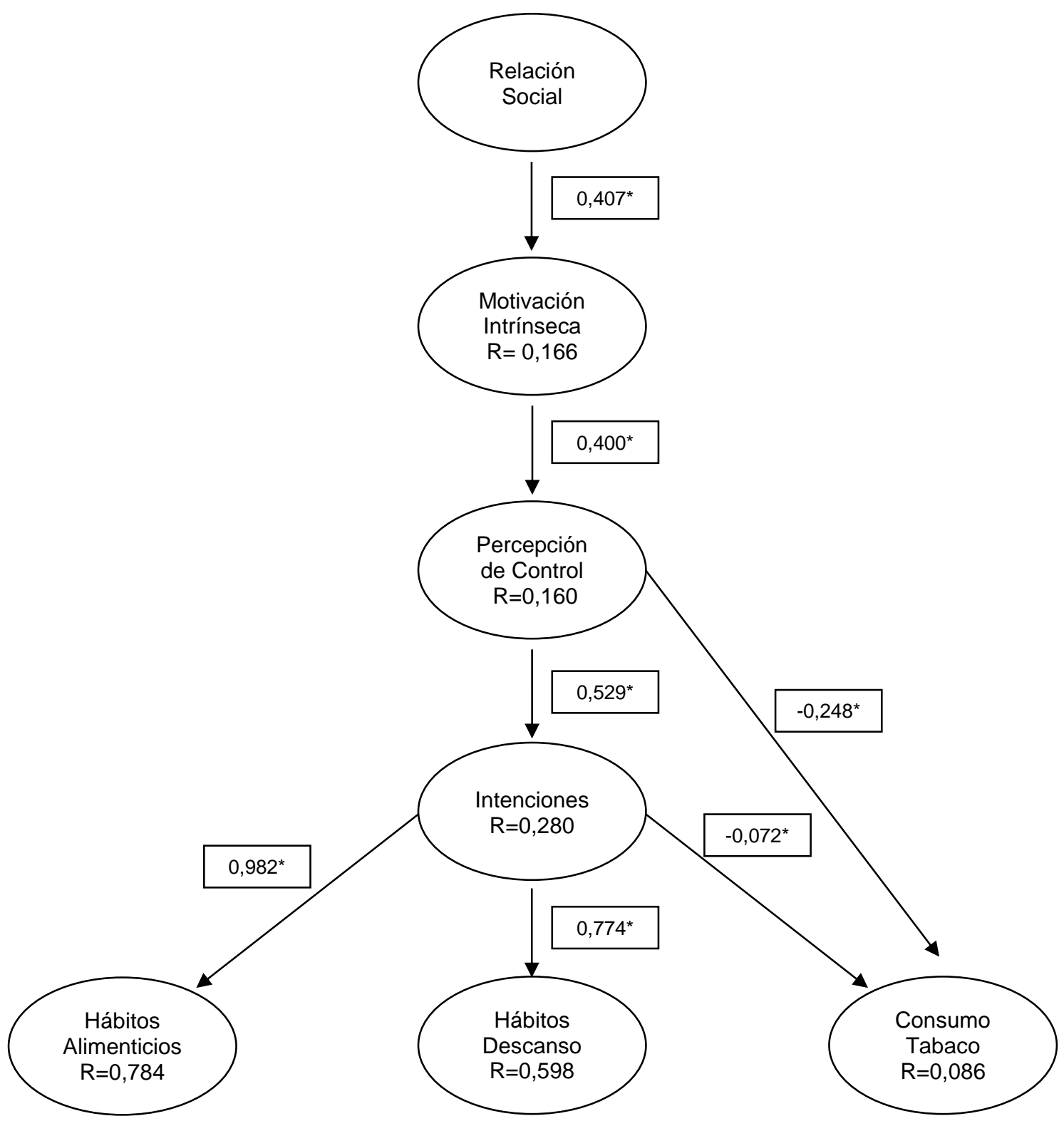

Figura 1. Modelo de ecuaciones estructurales la aplicación de lo Modelo Transcontextual al deporte veterano. Todos los pesos de regresión están estandarizados y son estadísticamente significativos.

\section{DISCUSIÓN}

Este trabajo tuvo como principal objetivo aplicar el Modelo Transcontextual de la motivación en la predicción de estilos de vida saludables de atletas veteranos. Los resultados del análisis de ecuaciones estructurales realizados mostraron el cumplimiento del mismo.

Existen pocas investigaciones, hasta la fecha, que tengan analizadas las relaciones propuestas fuera del ámbito de la educación física. La mayoría de los estudios del Modelo Transcontextual se han centrado en comprobar que la motivación desarrollada en los alumnos, con base en la autonomía en las clases de educación física, se puede transferir a la práctica de actividades físicas 
diversas en el contexto de ocio. Las investigaciones revelan también excelentes indicadores respecto a la autonomía dada por los familiares a los practicantes, en el sentido de ser un predictor para la adhesión a la práctica de actividad física o deportiva. La mayoría de los estudios llevados a cabo en el ámbito del deporte veterano se han centrado en comprobar algunas de las relaciones propuestas por la Teoría de la Autodeterminación. Este estudio va a permitir conocer en mayor medida el mecanismo motivacional que lleva a los atletas veteranos a mantenerse en la práctica físico-deportiva.

Los resultados descriptivos obtenidos en esta investigación, en las variables de la Teoría de la Autodeterminación, concretamente en las necesidades psicológicas básicas, determinan que la percepción de la necesidad psicológica básica de relación social ha sido la variable con más media obtenida, seguida de la percepción de la necesidad psicológica básica de autonomía y la percepción de la necesidad psicológica básica de competencia, asumiendo la misma tendencia en los estudios de Batista et al. (2016a; 2016b), y Batista et al. (2017a; 2017b).

En el dominio de la motivación, la motivación autónoma ha sido valorada de forma elevada, corroborando los estudios de Da Silva (2009), Hodge, Allen, y Smellie (2008), Ruiz-Juan y Zarauz-Sancho (2012), o Sancho y Ruiz-Juan (2015), Batista et al. (2016a; 2016b), Batista et al (2017a; 2017b). La motivación controlada ha obtenido valores más bajos, de acuerdo con Ruiz-Juan y ZarauzSancho, (2012), Batista et al. (2016a; 2016b), Batista et al (2017a; 2017b), que han encontrado valores moderados de motivación extrínseca y casi nulos de desmotivación. Dodd y Spinks (1995), concluyeron que los atletas veteranos presentaban los dos tipos de motivación, intrínseca y extrínseca.

En las variables de la Teoría del Comportamiento Planeado, las intenciones han sido la variable más valorada por los atletas veteranos. Este hallazgo se encuentra en relación a lo expuesto por Ajzen (1985), Courneya y Bobick (2000), Palmeira y Teixeira (2004) o Palmeira et al. (2007), donde las intenciones se presentan como la principal variable predictora de comportamientos, en la medida que, las intenciones reflejan la cantidad de esfuerzo o intensidad que un individuo va a invertir para perseguir los resultados futuros.

En las variables de estilos de vida saludables se han obtenido valoraciones elevadas en los hábitos alimenticios y de descanso y valoraciones más bajas en el consumo de tabaco, tal como los hallazgos de Batista et al. (2016a), lo que nos lleva a adoptar la idea de que los individuos altamente vinculados con el deporte $\mathrm{o}$ la actividad física asumen comportamientos relacionados con estilos de vida saludables y eliminan comportamientos que condicionan la salud.

Incidiendo ahora sobre el análisis de correlaciones, éste ha revelado que las variables percepción de autonomía, percepción de competencia, percepción de relación social, la variable motivación intrínseca, regulación integrada, regulación identificada, las variables actitudes, normas subjetivas, percepción de control, intenciones y hábitos alimenticios y de descanso se correlacionaban positiva y significativamente entre sí. Las variables regulación introyectada, regulación externa y desmotivación han revelado correlaciones negativas y en su mayoría 
significativas con las demás variables, exceptuando con la variable consumo de tabaco, con la cual se han obtenido correlaciones negativas y significativas. Se ha observado, por lo tanto, que las variables de la motivación autónoma (motivación intrínseca, regulación integrada y regulación identificada) han presentado una relación positiva con las necesidades psicológicas básicas, con las variables del comportamiento planeado y con las variables comportamentales de un estilo de vida saludable. A su vez, las variables que constituyen la motivación controlada (regulación introyectada y regulación externa) presentan una relación inversamente proporcional con las variables citadas anteriormente, con excepción de la variable comportamental prejudicial para la salud consumo de tabaco. De una forma global estas tendencias entre variables respetan el Modelo Transcontextual de Hagger et al. (2003), habiendo obtenido Batista et al. (2016a; 2016b) y Batista et al. (2017b) en sus estudios, resultados análogos.

Observaciones parecidas han sido obtenidas en otros estudios (Leyton, 2014; Pizarro, 2014; Hagger et al., 2014), donde han comprobado que el fomento de la motivación intrínseca favorecía, positivamente, el aumento de la satisfacción de las necesidades psicológicas básicas de autonomía y competencia, las formas de motivación más autodeterminadas y los hábitos alimenticios. La práctica de actividad física predice, de forma positiva y significativa, la alimentación equilibrada y el respeto al horario de las comidas y, sin evidenciar significancia estadística, los hábitos de descanso.

En nuestro estudio, las correlaciones positivas entre los hábitos saludables de descanso y nutricionales con la motivación autodeterminada, reflejan las tendencias apuntadas por otros autores (Prochaska, Spring y Nigg, 2008; García-Ubaque, 2011; Pizarro, 2014; Hagger et al., 2014), evidenciando conductas saludables integradas en el contexto del deporte veterano, paralelamente a una autodeterminación hacia la práctica. Los resultados obtenidos, favorecieron los niveles de motivación más autodeterminados en la realización de la práctica deportiva por el placer propio que ésta supone, incrementan así un estilo de vida saludable. Leyton et al. (2012) han observado que los niveles de motivación más autodeterminada presentaban una relación positiva con las variables de estilos de vida que realzan la salud.

De manera global, a raíz de los resultados encontrados, y atendiendo a la Teoría de la Autodeterminación (Deci y Ryan, 1980, 1985, 1991, 2000, 2012) en la cual se basa este trabajo, estos parecen respetar la tendencia igualmente encontrada por otros autores (Moreno, Moreno y Cervelló, 2007; DeFreese y Smith, 2013), que han demostrado la relación entre la práctica de actividades físico-deportivas y la motivación más autodeterminada, lo que, aparentemente, se observa en nuestro estudio, dado la tendencia de los deportistas encuestados para una base motivacional de comportamientos más autodeterminados, teniendo como consecuencia, el mantenimiento de estilos de vida saludables (Nutbeam, Aaro y Catford, 1989).

Pasando a la interpretación del modelo de ecuaciones estructurales obtenido en nuestro estudio, este ha revelado que la necesidad psicológica básica de relación social es un predictor de la motivación intrínseca. Este indicador respecta los hallados de Barkoukis Hagger, Labropoulos y Tsorbatzoudis (2010), donde la 
satisfacción de la necesidad psicológica básica de relaciones sociales ha sido un mediador en el contexto de la actividad física de ocio, aunque en nuestro estudio, no se encontró la competencia como factor predictor, al igual que estos autores.

A su vez, la motivación intrínseca ha revelado ser un predictor de la percepción de control comportamental. Hagger et al. (2003), apuntan que los tipos más autónomos de motivación en las prácticas de actividad física de ocio predicen positivamente las actitudes, así como la percepción de control comportamental.

Esta última variable ha sido predictora de las intenciones, al igual que propuso Ajzen (1985). Las variables de hábitos alimenticios y hábitos de descanso han sido predicho positivamente por las intenciones, y el consumo de tabaco ha sido predicho negativamente por las actitudes y por la percepción de control, asumiendo en nuestro estudio, un valor de mayor énfasis predictivo en la percepción de control seguido de las actitudes, respetando el modelo original propuesto por Ajzen (1985) con resultados similares que en los estudios de Courneya y Bobick (2000), Palmeira y Teixeira (2004) o Palmeira et al. (2007). Hagger y Chatzisarantis (2016), apuntan que la satisfacción global actúa como un mecanismo por lo cual la motivación autónoma en el contexto deportivo puede promover motivación autónoma fuera de ese contexto, siendo un excelente indicador de una conducta comportamental con vista a un pleno estado de salud por parte de los atletas veteranos.

A nuestro entender, la obtención de estos resultados aparentemente van al encuentro de una mayor percepción de la necesidad psicológica básica de relación social por parte de los atletas veteranos a medida que la edad va aumentando, dejando de ser determinantes la competencia percibida y la autonomía, ya que la experiencia acumulada a lo largo de los años les dará un soporte sólido para su comportamiento sea en el entrenamiento, o sea en la competición. La necesidad psicológica básica de relación social es el sentimiento de que se puede contar con la colaboración y aceptación de las personas consideradas importantes (Moreno-Murcia, et al., 2011) y es esencial para la adquisición de los reglamentos sociales (normas, reglas y valores), pues es a través de los vínculos con otros, como se desarrolla el aprendizaje (Deci y Ryan, 2000). Esta tendencia del nivel de satisfacción de las necesidades psicológicas básicas como mecanismos predictores de motivación y consecuentemente de bienestar y de estilos de vida saludables, se han mostrado de manera idéntica en practicantes de actividades físicas de ocio en practicantes con edades superiores (Leyton et al., 2012; Moutão, Alves y Cid, 2012; Leyton, 2014; Pizarro, 2014; Hagger et al., 2014; Batista et al., 2016a, 2016b, 2017b).

Como limitaciones de este estudio hay que señalar en primer lugar, que por el hecho de tratarse de un estudio correlacional, no se pueden establecer correlaciones causales aunque aporte un modelo explicativo, que permite una mayor y mejor comprensión de la relación conjunta existente entre las distintas variables analizadas. Se señala además el problema de modelos equivalentes que presentan la técnica de ecuaciones estructurales (Hersberger, 2006), que asume que el modelo presentado en este estudio no sería más que uno de los posibles modelos. 
En una perspectiva de mejoría para estudios futuros, el hecho de aplicar únicamente cuestionarios, seria interesante obtener información a través de otra metodología que permita una triangulación de los datos, a través de entrevistas, grupos focalizados, registros de frecuencia al entrenamiento, $u$ otras fuentes de información. Así, sería recomendable en el futuro la realización de estudios longitudinales y diseños experimentales a través de los cuales se pueda comprobar el efecto de determinadas intervenciones, como la aplicación de programas donde se trabajen las necesidades psicológicas básicas y la motivación, así como comprender la importancia de otras variables en la percepción de los atletas veteranos.

Como propuestas de aplicación práctica, el modelo de ecuaciones estructurales obtenido en esta investigación ha revelado que la necesidad psicológica básica de relación social es un predictor de la motivación intrínseca. Los resultados determinan la importancia que supone para los atletas veteranos el contexto de práctica deportiva, pues resulta crucial que sientan proximidad y apoyo por parte del entrenador. Es esencial que los atletas sientan apoyo desde el líder del entrenamiento y que éste esté disponible para ayudarlos. Otra estrategia para el desarrollo de relaciones sociales se basa en organizar tareas en las cuales los atletas puedan participar y se unan por un fin común. El traslado de experiencia desde los más a los menos experimentados es algo que tanto en el entrenamiento como en la competición debe ser una realidad. De este modo, el empleo de estrategias, particularmente en el entrenamiento, donde pueda haber un reparto de estrategias ante problemas propuestos podrá ser un hábito a implantar. El tiempo de ocio es esencial para el bienestar y la adopción de conductas de salud en cualquier individuo. Así será igualmente importante promover tiempo de ocio con el grupo de entrenamiento, a parte contexto deportivo que es donde normalmente interactúan.

La motivación intrínseca es un predictor de la percepción de control comportamental. Es importante que se trabaje con los atletas veteranos una base motivacional autónoma, en la medida en que esta conducirá las consecuencias deseadas y más adaptativas. Se debe promover una práctica cuyo objetivo sea la diversión y el placer de su vivencia.

Como variable predictora de las intenciones, la de mayor énfasis es la percepción de control. Las intenciones reflejan representaciones para estadios deseados, lo cual entendemos que una buena formulación de objetivos será una buena estrategia para alcanzar esos mismos objetivos. Naturalmente que en esta formulación de objetivos, será importante la percepción de control sobre los comportamientos, lo que obliga a un análisis de las obligaciones y horarios a cumplir de cada individuo, para que este consiga realizar los entrenamientos y/o competiciones que se proponen.

Las variables hábitos alimenticios y hábitos de descanso han sido predichas por las intenciones, sin embargo, el consumo de tabaco ha sido predicho negativamente por las intenciones, así como, por la percepción de control. Estas últimas, han generado como consecuencia un proceso adaptativo de carácter comportamental, hábitos saludables dónde cada atleta veterano se podrá sentir 
con ánimo, bienestar o felicidad, generando adaptaciones positivas cognitivas, afectivas y/ o comportamentales.

\section{CONCLUSIONES}

Teniendo como objetivo de estudio de esta investigación aplicar el Modelo Transcontextual de la motivación en la predicción de estilos de vida saludables de atletas veteranos, ha sido posible crear un modelo de ecuaciones estructurales que explican los estilos de vida saludables de atletas a partir de la integración de diversas teorías.

En conclusión, los resultados del estudio muestran que será determinante fomentar la necesidad psicológica básica de relación social, ya que ésta favorecerá la motivación intrínseca, promoviendo un mayor control comportamental sobre las intenciones de los practicantes, generando así hábitos alimenticios saludables, adecuados hábitos de descanso y menor consumo de tabaco.

\section{REFERENCIAS BIBLIOGRÁFICAS}

Adler A. (1929). The Practice and Theory of Individual Psychology. London: Routledge \& Kegan Paul.

American College of Sports Medicine (2010). Selected Issues for the Master Athlete and the Team Physician: A Consensus Statement. Medicine \& Science in Sports \& Exercise, 42(4), 820-833.

Ajzen, I. (2014). The theory of planned behavior is alive and well, and not ready to retire. Health Psychology Review. Advance online publication. DOI:10.1080/17437199.2014.883474

Ajzen, I. (1991). The Theory of Planned Behavior. Organizational Behavior and Human Processes, 50(2), 179-211. DOI: http://dx.doi.org/10.1016/0749-5978(91)90020-T

Ajzen, I. (1985). From intentions to actions: A theory of planned behavior. In J. Kuhl \& J. Beckmann (Eds.), Action-control: From cognition to behavior (pp. 11-39). Heidelberg, Germany: Springer. DOI: http://dx.doi.org/10.1007/978-3-642-697463_2

Albuquerque, F., Sousa, F., \& Martins, C. (2010). Validação das escalas de satisfação com a vida e afetos para idosos rurais. Psicologia, 41(1), 85-92.

Anderson, J., \& Gerbing, D. (1988). Structural equation modeling in practice: A review and recommended two-step approach. Phychological Bulletin, 103, 411-423. DOI: http://dx.doi.org/10.1037/0033-2909.103.3.411

Appel-Silva, M., Wendt, G., \& Argimon, I. (2010). A teoria da autodeterminação e as influências socioculturais sobre a identidade. Psciologia em Revista, 16(2), 351369.

Baker, J., Horton, S., \& Weir, P. (2010). The masters athlete: Understanding the role of sport and exercise in optimizing aging. New York: Routledge. DOI: http://dx.doi.org/10.4324/9780203885512

Batista, M., Jiménez, R., Leyton, M., Lobato, S., \& Aspano, M. (2016a). Adaptation and validation of the portuguese version of the healthy life styles questionnaire. Ponte - International Scientific Researches Journal, 72 (9), 145-158. ISSN: 0032-423x. DOI: http://dx.doi.org/10.21506/j.ponte.2016.9.11.

Batista, M., Jiménez, R., Honório, S., Petrica, J., \& Serrano, J. (2016b). Selfdetermination and life satisfaction: An exploratory study with veteran judo 
athletes. Revista de Artes Marciales Asiáticas, 11(2s), 90-91. DOI: 10.18002/rama.v11i2s.4187.

Batista, M., Jiménez, R., Lobato, S., Leyton, M., \& Aspano, M. (2017a). Diferenças em funcão do género das formas de motivação autodeterminada de atletas veteranos. Ágora para la Educación Física y el Deporte, 19(1), 35-51. DOI: https://doi.org/10.24197/aefd.1.2017.35-51.

Batista, M., Jiménez, R., Leyton, M., Aspano, M., \& Lobato, S. (2017b). Self-determined motivation and life satisfaction in Portuguese veterans athletes. Retos, 32, 124129. ISSN: Edición impresa: 1579-1726. Edición Web: 1988-2041.

Barkoukis, V., Hagger, M., Labropoulos, G., \& Tsorbatzoudis H. (2010). Extending the trans-contextual model in physical education and leisure-time contexts: examining the role of basic psychological need satisfaction. British Journal of Educacional Psychology, 80, 647-670. DOI: http://dx.doi.org/10.1348/000709910X487023

Bandura, A. (1982). Self-efficacy mechanism in human agency. American Psychologist, 37, 122-147. DOI: http://dx.doi.org/10.1037/0003-066X.37.2.122

Bentler, P. (1990). Comparative fit indexes in structural models. Psychological Bulletin, 107, 238-246. DOI: http://dx.doi.org/10.1037/0033-2909.107.2.238

Bollen, K., \& Long, J. (1993). Testing structural equation models. Newbury Park, CA: Sage.

Browne, M., \& Cudeck, R. (1993). Alternative ways of assessing model fit. In Bollen, K.A. \& Long, J.S. [Eds.] Testing structural equation models. Newbury Park, CA: Sage, $136-162$.

Carvalho, L., Avelar Rosa, B., \& Carvalho, A. (2014). Capitalização do valor educativo e social da Ética no Desporto no quadro da sua dimensão europeia e de definição de política de financiamento. Mafra: Instituto Luso-llírio para o Desenvolvimento Humano

Courneya, K., \& Bobick, T. (2000). Integrating the Theory of Planned Behavior with the Processes and States of Change in the Exercise Domain. Psychology of Sport and Exercise, 1, 41-56.

Cubo Delgado, S.; Martín Marin, B., \& Ramos Sanchez, J. (2011). Métodos de Investigación y Análisis de Datos en Ciências Sociales y de la Salud. Madrid: Pirâmide.

Da Silva, E. (2009). Motivação de atletas idosos na associação de veteranos de atletismo do Estado do Rio de Janeiro. Tesis Doctoral sin publicar. Universidad de Niterói, Rio de Janeiro, Brasil.

Deci, E, \& Ryan, R. (2012). Self-determination theory. In A. W. Kruglanski, P. A. M. Van Lange, \& E. T. Higgins (Eds.), Handbook of theories social psychology (pp. 416437). London: Sage. DOI: http://dx.doi.org/10.4135/9781446249215.n21

Deci, E., \& Ryan, R. (2000). The ,,,what"' and ",,why"'"' of goal pursuits: Human needs and the self-determination of behavior. Psychological Inquiry, 11, 227-268. DOI: http://dx.doi.org/10.1207/S15327965PLI1104_01

Deci, E., \& Ryan, R. (1991). A motivational approach to self: Integration in personality. In R. Dienstbier (Ed.), Nebraska symposium on motivation: Perspectives on motivation (pp. 237-288). Lincoln, NE: University of Nebraska Press.

Deci, E., \& Ryan, R. (1985). Intrinsic motivation and Self-determination in human behavior. New York: Plenum. DOI: http://dx.doi.org/10.1007/978-1-4899-2271-7

Deci, E., \& Ryan, R. (1980). The empirical exploration of intrinsic motivational processes. In L. Berkowitz (Ed.), Advances in experimental social psychology (pp. 39-80). New York: Academic Press. DOI: http://dx.doi.org/10.1016/S0065-2601(08)601306

DeFreese, J., \& Smith, A. (2013). Teammate social support, burnout, and selfdetermined motivation in collegiate athletes. Psychology of Sport and Exercise, 14, 258-265. DOI: http://dx.doi.org/10.1016/j.psychsport.2012.10.009 
Diener, E. (2013). The Remarkable Changes in the Science. Perspectives on Psychological Science, 8(6), 663-666.

Diener, E. (1994). Assessing subjective well-being: progress and opportunities. Social Indicators Research, 31(2), 103-157. DOI: http://dx.doi.org/10.1007/BF01207052

Diener, E., Suh, E., Lucas, R., \& Smith, H. (1999). Subjective well-being: Three decades of progress. Psychological Bulletin, 125, 276-302. DOI: http://dx.doi.org/10.1037/0033-2909.125.2.276

Diener, E., Emmons, R., Larsen, R., \& Griffin, S. (1985). The satisfaction with life scale. Journal of Personality Assessment, 49(1), 71-75. DOI: http://dx.doi.org/10.1207/s15327752jpa4901_13

Dodd, J., \& Spinks, W. (1995) Motivations to engage in masters sport. ANZALS Leisure Research Series, 2, 61-75.

García-Ubaque, J. (2011). Hábitos saludables de los trabajadores de una institucion hospitalaria pública de alta complejidad en Bogotá. Tesis Doctoral. Bogotá. Universidad Nacional de Colombia.

González-Cutre, D., Sicilia, À., Beas-Jiménez, M., \& Hagger, M. (2014). Broadening the trans-contextual model of motivation: A study with Spanish adolescentes. Scandinavian Journal of Medicine Science in Sports, 24, 306-319 DOI: https://doi.org/10.1111/sms.12142

Gurtner, J., Gulfi, A., Genoud, P., Trindade, B., \& Schumacher, J. (2012). Learning in multiple contexts: are there intra-, cross- and transcontextual effects on the learner's motivation and help seeking? European Journal Psychological Educación, 27, 213-225. DOI: http://dx.doi.org/10.1007/s10212-011-0083-4

Hagger, M. S., \& Chatzisarantis, N. L. D. (2016). The Trans-Contextual Model of Autonomous Motivation in Education: Conceptual and Empirical Issues and MetaAnalysis. Review of Educational Research, 86(2), 360-407. DOI: $10.3102 / 0034654315585005$

Hagger, M., Hardcastle, S., Chater, A., Mallett, C., Pal, S., \& Chatzisarantis, N. (2014). Autonomous and controlled motivational regulations for multiple health-related behaviours: between- and within-participants analyses. Health Psychology \& Behavioural Medicine, 2, 565 - 601.

Hagger, M. S., \& Chatzisarantis, N. L. D. (2012). Transferring motivation from educational to extramural contexts: A review of the trans-contextual model. European Journal of Psychology of Education, 27(1), 195-212. DOI:10.1007/s10212-011-0082-5

Hagger, M., \& Chatzisarantis, N. (2009a). Assumptions in research in sport and exercise psychology. Psychology of Sport and Exercise, 10, 511-519. DOI:10.1016/j.psychsport.2009.01.004

Hagger, M., \& Chatzisarantis, N. (2009b). Integrating the theory of planned behaviour and self-determination theory in health behaviour: A meta-analysis. British Journal of Health Psychology, 14, 275-302. DOI:10.1348/135910708X373959

Hagger, M., Anderson, M., Kyriakaki, M., \& Darkings, S. (2007a). Aspects of identity and their influence on intentional behaviour: comparing effects for three health behaviours. Personality and Individual Differences, 42, 355-367.

Hagger, M., Chatzisarantis, N., Culverhouse, T., \& Biddle, S. (2003). The processes by which perceived autonomy support in physical education promotes leisure-time physical activity intentions and behavior: A trans-contextual model. Journal of Educational Psychology, 95, 784-795. DOI:10.1037/00220663.95.4.784

Hershberger, S. (2006). The problem of equivalent structural models. In G.R. Hancock, \& R.O. Mueller (Eds.), Structural equation modeling: a course (pp.13-42). Greenwich, CT: Information Age Publishing.

Hodge, K., Allen, J., \& Smellie, L. (2008). Motivation in masters sport: Achievement and social goals. Psychology of Sport and Exercise, 9 (2), pp. 157-176. DOI: http://dx.doi.org/10.1016/j.psychsport.2007.03.002 
Hu, L., \& Bentler, P. M. (1999). Cutoff criteria for fit indexes in covariance structure analysis: Conventional criteria versus new alternatives. Structural Equation Modeling, 6, 1-55. DOI:10.1080/10705519909540118

Instituto do Desporto de Portugal (2011). Estatísticas do Desporto de 1996 a 2009. Lisboa: Instituto do Desporto de Portugal, IP.

Iso-Ahola, S., \& St.Clair, B. (2000). Toward a theory of exercise motivation. Quest, 52: 131-147. DOI: http://dx.doi.org/10.1080/00336297.2000.10491706

Leyton, M., Jiménez, R., Domínguez, F., \& Corzo, H. (2012). Análisis correlacional entre la teoría de la autodeterminación y variables de estilos de vida saludables: descanso, tabaco y alimentación. IV Congreso Internacional de Ciencias del Deporte y la Educación Física. (VIII Seminario Nacional de Nutrición, Medicina y Rendimiento Deportivo) Pontevedra, España. 2012; 10-12.

Leyton, M. (2014). Aplicación de un programa de ejercicio físico para optimizar la motivación y los hábitos saludables en los adultos. Tesis doctoral. Cáceres: Universidad de Extremadura.

Lonsdale, C., Hodge, K., \& Rose, E. A. (2008). The Behavioral Regulation in Sport Questionnaire (BRSQ): Instrument Development and Initial Validity Evidence. Journal of Sport \& Exercise Psychology, 30, 323-355. DOI: http://dx.doi.org/10.1123/jsep.30.3.323

Lopes, M. (2012). Hábitos de vida dos adolescentes: Género, ano de escolaridade e prática de atividade física. Dissertação de Mestrado, Faculdade de Motricidade Humana, Universidade Técnica de Lisboa, Lisboa.

Mata, J., Silva, M., Vieira, P., Carraça, E., Andrade, A., Coutinho, S., Sardinha, L., \& Teixeira P. (2009). Motivational "spill-over" during weight control: increased selfdetermination and exercise intrinsic motivation predict eating self-regulation. Health Psychology, 28, 709-716. DOI: http://dx.doi.org/10.1037/a0016764

Marsh, H., Richards, G., Johnson, S., Roche, L., \& Tremayne, P. (1994). Physical Self Description Questionnaire: Psychometric properties and a multitrait-multimethod analysis of relations to existing instruments. Journal of Sport and Exercise Psychology, 16, 270-305. DOI: http://dx.doi.org/10.1123/jsep.16.3.270

McDonald, R., \& Marsh, H. (1990). Choosing a multivariate model: Noncentrality and goodness of fit. Psychological Bulletin, 107, 247-255. DOI: http://dx.doi.org/10.1037/0033-2909.107.2.247

Monteiro, D., Moutão, J., Baptista, P., \& Cid, L. (2014). Clima motivacional, regulação da motivação e perceção de esforço dos atletas no futebol. Motricidade, 10(4), 94104. DOI: http://dx.doi.org/10.6063/motricidade.10(4).3453

Moreno, J., Moreno, R., \& Cervelló, E. (2007). El autoconcepto físico como predictor de la intención de ser fisicamente activo. Psicología y Salud, 17, 261-267.

Moreno, J., Hernández, A., \& González-Cutre, D. (2009). Complementando la teoría de la autodeterminación con las metas sociales: un estudio sobre la diversión en educación física. Revista Mexicana de Psicología, 26(2), 213-222.

Moreno-Murcia, J., Marzo, J., Martínez-Galindo, C., \& Marín, L. (2011). Validación de la Escala de "Satisfacción de las Necesidades Psicológicas Básicas" y del Cuestionario de la "Regulación Conductual en el Deporte" al contexto español. International Journal of Sport Science, 7, 355-369.

Moutão, J., Cid, L., Alves, J., Leitão, J., \& Vlachopoulos, S. (2012). Validation of the Basic Psychological Needs in Exercise Scale in a Portuguese Sample. The Spanish Journal of Psychology, 15(1), 399-409. DOI: http://dx.doi.org/10.5209/rev_SJOP.2012.v15.n1.37346

Moutão, J., Alves, S., \& Cid, L. (2012). Contributo da teoria da autodeterminação na predição da vitalidade e adesão ao exercício físico. Gymnasium 3(1), 13 - 34. 
Neto, F. (1993). The Satisfaction with Life Scale: Psychometrics Properties in an Adolescent Sample. Journal of Youth and Adolescence, 22(2), 125-134. DOI: http://dx.doi.org/10.1007/BF01536648

Nutbeam, D., Aaro, L., \& Catford, J. (1989). Understanding children"s health behaviour: The implication for health promotion for young people. Social Science and Medicine, 29 (3): 317-325.

Odgen C, \& Carrol M. (2010). Prevalence of overweight, obesity, and extreme obesity among adults: United States, trends 1960-1962 through 2007-2008. National Center for Health Statistics - Division of Health and Nutrition Examination Surveys, 1-5.

Orbell, S., Hagger, M. S., Brown, V., \& Tidy, J. (2006). Comparing two theories of health behavior: A prospective study of non-completion of treatment following cervical cancer screening. Health Psychology, 25, 604-615. DOI:10.1037/02786133.25.5.604

Palmeira, A., \& Teixeira, P. (2004). Validação Preliminar De Um Instrumento De Avaliação Da Teoria Do Comportamento Planeado No Controlo De Peso. Lisboa. Edited by: Ribeiro JLP.

Palmeira, A., Teixeira, P., Branco T., Martins, S., Minderico, C., Barata, J., Serpa, S., \& Sardinha, L. (2007). Predicting short-term weight loss using four leading health behavior change theories. International Journal of Behavioral Nutrition and Physical Activity, 4, 14. DOI: http://dx.doi.org/10.1186/1479-5868-4-14

Pavey L.J., \& Sparks P. (2010). Autonomy and reactions to health-risk information. Psychology Health, 25, 855-872.

Pires, A., Cid, L., Borrego, C., Alves, J., \& Silva, C. (2010). Validação preliminar de um questionário para avaliar as necessidades psicológicas básicas em Educação Física. Motricidade, 6(1), 33-51. DOI: http://dx.doi.org/10.6063/motricidade.6(1).157

Pizarro P. (2014). Análisis de la motivación en la práctica de actividad física y ejercicio físico. Trabajo de Master. Cáceres: Universidad de Extremadura.

Prochaska, J., Spring, B., \& Nigg C. (2008). Multiple health behavior change research: An introduction and overview. The American Journal of Preventive Medicine, 46, 181-188. DOI: http://dx.doi.org/10.1016/j.ypmed.2008.02.001

Riebe, D., Franklin, B., Thompson, P., Garber, Carol, E., Whitfield, G., Magal, M., \& Pescatello, L. (2015). Updating ACSM's Recommendations for Exercise Preparticipation Health Screening. Medicine \& Science in Sports \& Exercise, 47(11), 2473-2479). DOI: 10.1249/MSS.0000000000000664

Rodríguez-Marín, J., \& García-Hurtado, J. (1995). Estilo de vida y salud. En: Latorre, J. M., editor. Ciencias psicosociales aplicadas II. Madrid: Síntesis.

Ruiz-Juan, F., Isorna, M., Ruiz-Risueño, J., \& Vaquero-Cristóbal, R. (2014). Consumo e ingesta de alcohol en españoles mayores de 16 años y su relación con la actividad físico-deportiva, la familia y el consumo de tabaco. Revista lberoamericana de Psicología del Ejercicio y el Deporte, 9(2), 339-372.

Ruiz-Juan, F., \& Zarauz-Sancho, A. (2012). Predictor variables of motivation in Spanish master athletes. Journal of Human Sport and Exercise, 7(3), 617-628. DOI: http://dx.doi.org/10.4100/jhse.2012.73.02

Ryan, R. (1995). Psychological needs and the facilitation of integrative processes. Journal of Personality, 63(3), 397-427. DOI: http://dx.doi.org/10.1111/j.14676494.1995.tb00501.x

Simões, F., \& Alarcão, M. (2013). Satisfação de necessidades psicológicas básicas em crianças e adolescentes: adaptação e validação da ESNPBR. Psicologia: Reflexão e Crítica, 26(2), 261-269. DOI: http://dx.doi.org/10.1590/S010279722013000200006 
Siqueira, M., \& Padovam, V. (2008). Bases teóricas de bem-estar subjetivo, bem-estar psicológico e bem-estar no trabalho. Psicologia: Teoria e Pesquisa, 24(2), 201209. DOI: http://dx.doi.org/10.1590/S0102-37722008000200010

Telama, R., Yang, X., Leskinen, E., Kankaanpa, A., Hirvensalo, M., Tammelin, T., Viikari, J. \& Raitakari, O. (2014). Tracking of Physical Activity from Early Childhood through Youth into Adulthood. Medicine and Science In Sports And Exercise. 46(5), 955-962. DOI: http://dx.doi.org/10.1249/MSS.0000000000000181

Vallerand, R. (2015). The psychology of passion: A dualistic model. Oxford University Press. DOI: http://dx.doi.org/10.1093/acprof:oso/9780199777600.001.0001

Vallerand, R. (2007). A hierarchical model of intrinsic and extrinsic motivation for sport and physical activity. In M. S. Hagger, \& N. Chatzissaratis (Eds.), Intrinsic motivation and self-determination in exercise and sport (pp. 255-280). Champaign: Human Kinetics.

Vallerand, R. (2001). A hierarchical model of intrinsic and extrinsic motivation in sport and exercise. In G.C. Roberts (Ed.), Advances in motivation in sport and exercise (pp. 263-320). Champaign, IL: Human Kinetics.

Vallerand, R. (1997). Toward a hierarchical model of intrinsic and extrinsic motivation. In M. Zanna (Ed.), Advances in experimental social psychology, pp. 271-360. New York: Academic Press.

Veblen, T. (1994). The Theory of the Leisure Class: An Economic Study of Institutions. Penguin twentiethcentury classics. New York: Penguin Books.

Veja, A., Aramendi, P., Buján, M., \& Garín, S. (2015). La educación para la salud en la ESO: Aportaciones de un estudio sobre el País Vasco. Educación XXI, 18(1): 167188. DOI: $10.5944 /$ educXX1.18.1.12316

Vlachopoulos, S., \& Michailidou, S. (2006). Development and initial validation of a measure of autonomy, competence, and relatedness in exercise: The basic psychological needs in exercise scale. Measurement in Physical Education and $\begin{array}{lll}\text { Exercise } \quad \text { Science, 10(3), 179-201. DOI: } & \end{array}$ http://dx.doi.org/10.1207/s15327841mpee1003_4

Weber, M. (1946). Essays in sociology. New York: Oxford University Press.

Wold, B. (1995). Health-Behaviour in schoolchildren: A WHO crossnational Survey. Resource Package Questions 1993-4. Norway: University of Bergen.

World Health Organization (2016). World Health Statistics 2016: Monitoring health for the SDGs. Geneva: WHO.

World Health Organization (2002). The European Healyh Report 2002. Copenhagen: WHO Regional Office for Europe.

Zarauz-Sancho, A., \& Ruiz-Juan, F. (2013). Motivaciones de los maratonianos según variables socio-demográficas y de entrenamiento. Retos, 24, 50-56.

Zarauz-Sancho, A., \& Ruiz-Juan, F. (2014). Análisis de la motivación en el atletismo: un estudio con veteranos. Universitas Psychologica, 13(2), 501-515.

Zarauz-Sancho, A., \& Ruiz-Juan, F. (2015). Factores determinantes de la motivación en atletas veteranos españoles. Revista Latinoamericana de Psicología, 47(1), 3442. DOI: http://dx.doi.org/10.1016/S0120-0534(15)30004-2

Zarauz-Sancho, A., \& Ruiz-Juan, F. (2016). Motivación, satisfacción, percepción y creencias sobre las causas del éxito en atletas veteranos españoles. Revista Iberoamericana de Psicología del Ejercicio y el Deporte, 11(1), 37-46. ISSN 18868576.

Silva, S., Dias, C., \& Fonseca, A. (2011). Competências psicológicas em atletas veteranos. Revista Brasileira de Cineantropometria e Desempenho Humano, 13(5), 404-407. DOI: 10.5007/1980-0037.2011v13n5p404. 
Rev.int.med.cienc.act.fís.deporte - vol. 19 - número 75 - ISSN: 1577-0354

Referencias totales / Total references: 95 (100\%)

Referencias propias de la revista/Journal's own references: $0(0 \%)$

Rev.int.med.cienc.act.fís.deporte - vol. 19 - número 75 - ISSN: 1577-0354 